\title{
Magnetic properties and the phase diagram of $\mathrm{La}_{1-x} \mathrm{Sr}_{x} \mathrm{MnO}_{3}$ for $x \leqslant 0.2$
}

\author{
M Paraskevopoulos $\dagger$, F Mayr $\dagger$, J Hemberger $\dagger$, A Loidl $\dagger$, R Heichele $\ddagger$, \\ D Maurerł, V Müller $\ddagger$ A A Mukhin§ and A M Balbashov\| \\ $\dagger$ Experimentalphysik V, Elektronische, Korrelationen und Magnetismus, Institut für Physik, \\ Universität Augsburg, D-86159 Augsburg, Germany \\ ‡ Experimentalphysik III, Institut für Physik, Universität Augsburg, D-86159 Augsburg, Germany \\ $\S$ Moscow Power Engineering Institute, 105835 Moscow, Russia \\ || General Physics Institute of the Russian Academy of Sciences, 117942 Moscow, Russia
}

\begin{abstract}
We report on susceptibility, magnetization, magnetoresistance, and ultrasonic studies of $\mathrm{La}_{1-x} \mathrm{Sr}_{x} \mathrm{MnO}_{3}$ for $x \leqslant 0.2$. The magnetic susceptibilities in the different high-temperature paramagnetic phases are studied in detail. We provide experimental evidence for the occurrence of short-range ferromagnetic order within the paramagnetic phase. Furthermore, we can exclude the possibility of electronic phase separation for $x<0.1$. Variable-range-hopping conductivity dominates in the paramagnetic and magnetically ordered phases. From the anomalies at the structural and magnetic phase transitions, we arrive at a phase diagram which shows a ferromagnetic and insulating ground state for $0.1 \leqslant x \leqslant 0.15$, followed by a canted antiferromagnetic phase at higher temperatures. The ferromagnetic transition is strongly coupled to a structural transition from a Jahn-Teller-distorted phase to a pseudocubic orthorhombic phase and is accompanied by the appearance of large positive magnetoresistance effects. We interpret the rich phase diagram close to $x=1 / 8$ in terms of orbital ordering, due to an interplay of superexchange interactions and Jahn-Teller distortions.
\end{abstract}

\section{Introduction}

Almost half a century ago, Jonker and van Santen [1] demonstrated that the manganite perovskites $\left(\mathrm{La}^{3+} \mathrm{Mn}^{3+} \mathrm{O}_{3}^{2-}\right)_{1-x}\left(\mathrm{~A}^{2+} \mathrm{Mn}^{4+} \mathrm{O}_{3}^{2-}\right)_{x}$ reveal an interesting and puzzling phase diagram $[1,2]$. The close correlation of electrical and magnetic properties via an interplay of Mn-O-Mn superexchange (SE) interactions with Zener's double exchange (DE) [3] has been established by de Gennes [4]. An increasing concentration of mobile holes drives the insulating (I) and antiferromagnetic (AFM) structure via a canted AFM (CA) structure to a metallic (M) and ferromagnetic (FM) ground state [4]. An overwhelming interest in the manganites arose due to the observation of negative colossal magnetoresistance (CMR) close to $x \simeq 0.3[5,6]$. These CMR effects at the FM phase transition were explained within an extended double-exchange model [7] and also in models taking strong electron-phonon coupling into account $[8,9]$. Subsequently it was realized that the rich phase diagram observed in various manganite systems can only be explained considering additional degrees of freedom, such as Jahn-Teller (JT) distortions, [8,9] electronic correlations, and charge and orbital order [10-13].

An early structural phase diagram was presented by Bogush et al [14]. On decreasing the temperature, pure $\mathrm{LaMnO}_{3}$ reveals the following sequence: rhombohedral (R), orthorhombic 
$(\mathrm{O})$, and finally another orthorhombic structure $\left(\mathrm{O}^{\prime}\right)$. In the $\mathrm{O}$ phase the three octahedral $\mathrm{Mn}-\mathrm{O}$ bond lengths are almost equal, while in the JT-distorted $\mathrm{O}^{\prime}$ phase these bond lengths become anisotropic. An electronic phase diagram for $\mathrm{La}_{1-x} \mathrm{Sr}_{x} \mathrm{MnO}_{3}(x \leqslant 0.5)$ has been published by Urushibara et al [15]. That the $(x, T)$ phase diagram of $\mathrm{La}_{1-x} \mathrm{Sr}_{x} \mathrm{MnO}_{3}$ for low doping concentrations $(x<0.2)$ is even more complex has been established by Kawano et al [16], Yamada et al [17], and Zhou et al [18]. Guided by the classical conjectures of competing DE and SE interactions [4], in producing these phase diagrams it has been explicitly assumed that the regime where the resistivity decreases with decreasing temperature $(\mathrm{d} \rho / \mathrm{d} T>0)$ is metallic and reveals a simple FM spin arrangement, and that the low-temperature I ground state $(\mathrm{d} \rho / \mathrm{d} T<0)$ exhibits a CA structure for $x \leqslant 0.15$. Even now, this controversy has not been resolved. On the basis of neutron scattering results for $\mathrm{La}_{0.85} \mathrm{Sr}_{0.15} \mathrm{MnO}_{3}$, VasiliuDoloc et al [19] report on a canted antiferromagnetic ground state which is followed by a FM phase at elevated temperatures. For strontium concentrations $x=0.125$, Endoh et al [20] propose the existence of a FM insulator at low temperatures followed by a FM metal at elevated temperatures. It should also be noted that phase-separation models exist for the CA phase, which is believed to be unstable against separation into FM and metallic droplets embedded in a purely AFM and insulating phase. And indeed, neutron scattering results on $\mathrm{La}_{1-x} \mathrm{Ca}_{x} \mathrm{MnO}_{3}$, for Ca concentrations $x=0.05$ and 0.08 , were interpreted in terms of magnetic droplets [21].

On the basis of systematic magnetic susceptibility, magnetization, magnetoresistance (MR), and preliminary ultrasonic experiments on single-crystalline $\mathrm{La}_{1-x} \mathrm{Sr}_{x} \mathrm{MnO}_{3}$, we provide experimental evidence that all these phase diagrams are incorrect for $x \leqslant 0.2$. We construct a new phase diagram which documents the prime importance of SE interactions and orbital order in the lightly doped regime. In addition, from an analysis of the anisotropy of the magnetization we provide experimental evidence against electronic phase separation in $\mathrm{La}_{1-x} \mathrm{Sr}_{x} \mathrm{MnO}_{3}$ for $x<0.1$.

\section{Experimental details}

Single crystals of $\mathrm{La}_{1-x} \mathrm{Sr}_{x} \mathrm{MnO}_{3}$ with concentrations $0 \leqslant x \leqslant 0.2$, were grown by the floating-zone method with radiation heating. $\mathrm{Raw} \mathrm{La}_{2} \mathrm{O}_{3}, \mathrm{SrCO}_{3}$, and $\mathrm{Mn}_{3} \mathrm{O}_{4}$ chemicals of high purity (not less than $99.99 \%$ ) were used for ceramic rod preparation. 0.5 at. $\% \mathrm{Mn}_{3} \mathrm{O}_{4}$ excess concentration was used in order to compensate for Mn losses due to evaporation during the melting procedure. The initial synthesis of the composition was provided by annealing of the mixed chemical powder at about $1200{ }^{\circ} \mathrm{C}$ for $24 \mathrm{~h}$. After the feed rods were pressed, they were sintered at $1350{ }^{\circ} \mathrm{C}$ for $24 \mathrm{~h}$. The single crystals with $x \leqslant 0.075$ were grown in an $\mathrm{Ar}$ atmosphere, while for $x \geqslant 0.1$ an air atmosphere was used. The typical crystal growth speed was $7-10 \mathrm{~mm} \mathrm{~h}^{-1}$ using a rotation speed of crystal and feed rod of approximately 60 revolutions per minute. The typical growth direction was [110]. In order to obtain crack-free crystals, they were finally annealed at $1300{ }^{\circ} \mathrm{C}$ for several days.

$\mathrm{X}$-ray powder diffraction measurements revealed single-phase crystals. The rocking curves for the (110) plane showed halfwidths in the range $30^{\prime}-80^{\prime}$. The misorientation of the sub-blocks in the cross-section of the crystal did not exceed $1^{\circ}$ with respect to the [110] direction. Two-dimensional x-ray TV topography of the crystals indicated a twin structure for Sr concentrations $0 \leqslant x \leqslant 0.175$ for most of the single crystals. However, in favourable cases some of the small crystals, e.g. one with $x=0.05$, revealed a single domain only.

The magnetic susceptibility and magnetization were measured using an Oxford ac susceptometer in fields up to $140 \mathrm{kOe}$ and temperatures $1.5 \mathrm{~K}<T<300 \mathrm{~K}$, and a Quantum Design dc SQUID magnetometer (50 kOe, $1.5 \mathrm{~K}<T<800 \mathrm{~K}$ ). The magnetoresistance 
was measured with a standard four-probe technique in an Oxford cryostat and in fields up to $140 \mathrm{kOe}$.

Ultrasonic investigations were performed on larger single crystals some $\mathrm{mm}$ in length and with $\mathrm{Sr}$ concentrations of $x=0.05$ and $x=0.15$. The crystal orientation was determined by Laue $x$-ray diffraction. After cutting, pairs of parallel surfaces on each sample were polished to optical flatness. Longitudinal and transverse ultrasonic waves were generated and received using overtone $\mathrm{X}$ - and $a c$-cut quartz transducers which were carefully bonded to the sample with polysulphide liquid polymers. A high-resolution sampled-continuous-wave spectrometer [22] was used to perform temperature-dependent attenuation and sound velocity measurements at frequencies between 10 and $30 \mathrm{MHz}$. In order to avoid crystal damage due to thermal stress and to structural changes caused by the phase transitions, the samples were cooled down from ambient temperatures to $4 \mathrm{~K}$ and warmed up again quite slowly, the temperature change rate never exceeding $1 \mathrm{~K} \mathrm{~min}^{-1}$.

\section{Results and discussion}

\subsection{Magnetic properties}

3.1.1. Paramagnetic susceptibility. So far, not much attention has been paid to the behaviour of the high-temperature susceptibilities, with a direct correlation of the CurieWeiss temperatures and paramagnetic (PM) moments with structural properties. Töpfer and Goodenough [23] reported susceptibility measurements between $T_{C}$ and $600 \mathrm{~K}$ for $\mathrm{LaMnO}_{3+\delta}$ $(0 \leqslant \delta \leqslant 0.08)$. The $\chi^{-1}$-curves revealed significant non-linearities and effective moments considerably higher than the spin-only values of the manganese ions. These enhanced PM moments were ascribed to superparamagnetic clusters. Such 'giant' spins are often observed in Pauli paramagnets doped with $3 \mathrm{~d}$ ions (e.g. Fe in $\mathrm{Pd}, \mathrm{Cu}$, etc). Figure 1 shows the inverse susceptibility for a series of $\mathrm{La}_{1-x} \mathrm{Sr}_{x} \mathrm{MnO}_{3}$ samples for temperatures $T_{m} \leqslant T \leqslant 800 \mathrm{~K}$ $\left(T_{m}=T_{\mathrm{CA}}\right.$ for $x<0.15$ and $T_{m}=T_{C}$ for $\left.x \geqslant 0.15\right)$. The structural phase transitions $T_{\mathrm{OO}^{\prime}}$ $(x=0: 760 \mathrm{~K} ; x=0.05: 550 \mathrm{~K}$; and $x=0.125: 275 \mathrm{~K})$ and $T_{\mathrm{RO}}(x=0.125: 450 \mathrm{~K})$ can be clearly identified. The inverse susceptibility in the $\mathrm{O}^{\prime}$ phase for $x=0$ and $x=0.05$ is almost Curie-Weiss-like (CW-like). Strong deviations from a CW law appear in the rhombohedral phases for $x=0.125$ and $x=0.2$.

Figure 1 also demonstrates that the different structural phases are characterized by significantly different $\mathrm{CW}$ constants $\Theta$. A summary of the $\mathrm{CW}$ constants as a function of strontium concentration $x$ for the different structural phases $\left(\mathrm{O}, \mathrm{O}^{\prime}, \mathrm{R}\right)$ is given in table 1 and plotted

Table 1. The paramagnetic Curie-Weiss temperature $\Theta$ and effective magnetic moment $\mu_{\text {eff }}$ as extracted from the Curie-Weiss part of the $1 / \chi(T)$ curves for various $\mathrm{La}_{1-x} \mathrm{Sr}_{x} \mathrm{MnO}_{3}$ compounds. We have also included the calculated magnetic moment $\mu_{s}$ (spin only).

\begin{tabular}{|c|c|c|c|c|c|c|c|}
\hline \multirow[b]{2}{*}{$x$} & \multicolumn{2}{|c|}{$\mathrm{R}$ phase } & \multicolumn{2}{|c|}{ O phase } & \multicolumn{2}{|c|}{$\mathrm{O}^{\prime}$ phase } & \multirow{2}{*}{$\frac{\text { Spin-only value }}{\mu_{s}\left(\mu_{B}\right)}$} \\
\hline & $\mu_{e f f}\left(\mu_{B}\right)$ & $\Theta(\mathrm{K})$ & $\mu_{e f f}\left(\mu_{B}\right)$ & $\Theta(\mathrm{K})$ & $\mu_{e f f}\left(\mu_{B}\right)$ & $\Theta(\mathrm{K})$ & \\
\hline 0 & & & 4.98 & 198 & 5.10 & 66 & 4.90 \\
\hline 0.05 & & & 5.08 & 237 & 5.63 & 99 & 4.85 \\
\hline 0.075 & & & & & 5.93 & 110 & 4.82 \\
\hline 0.1 & & & 5.50 & 186 & 6.21 & 156 & 4.79 \\
\hline 0.125 & 4.76 & 336 & 5.89 & 233 & 6.61 & 190 & 4.77 \\
\hline 0.15 & 4.59 & 367 & 6.16 & 252 & & & 4.74 \\
\hline 0.2 & 4.66 & 384 & & & & & 4.69 \\
\hline
\end{tabular}




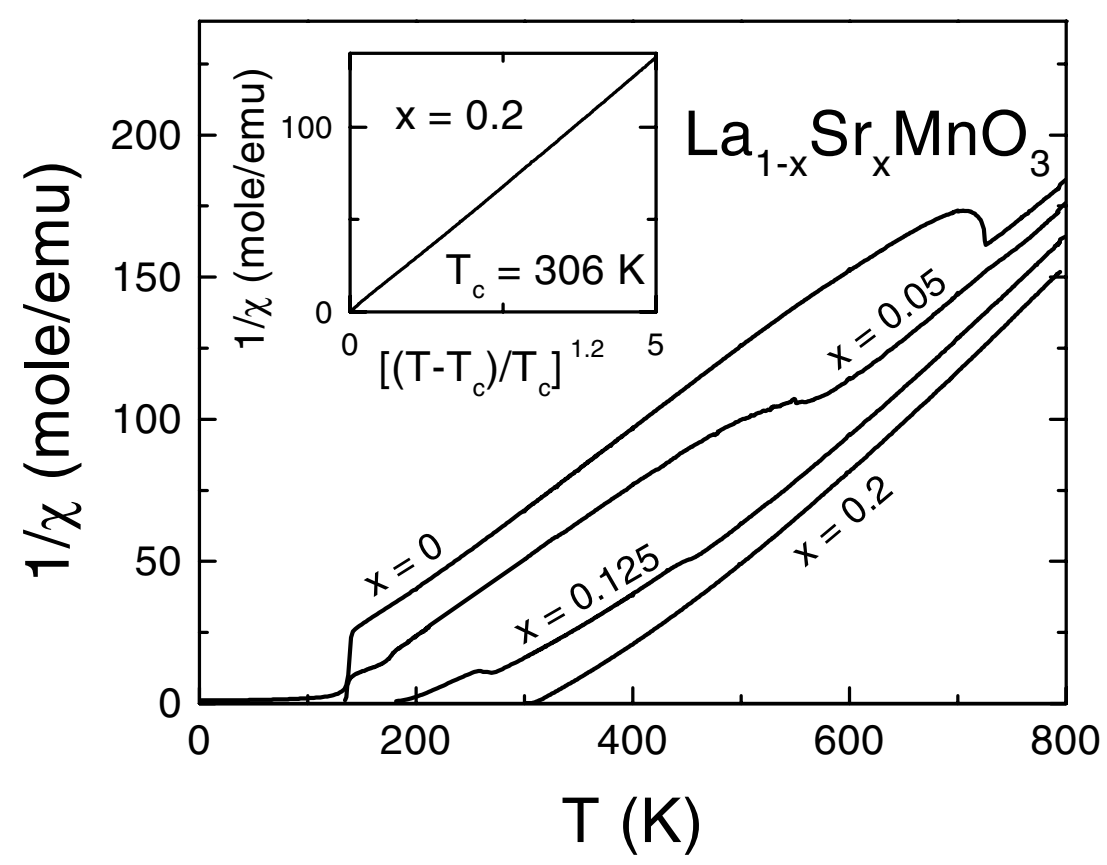

Figure 1. The temperature dependence of the inverse magnetic susceptibility $\chi^{-1}$ for various $\mathrm{Sr}$ concentrations. In the inset we show a fit of $\chi^{-1}$ for $x=0.2$, which revealed a critical exponent of $\gamma=1.2$ (see the text).

in figure 2(a). In the case of the occurrence of strong non-linearities, we analysed the hightemperature and low-temperature regimes separately (e.g. for $x=0.2, \chi^{-1}$ was analysed for $600 \mathrm{~K} \leqslant T \leqslant 800 \mathrm{~K}$ and $350 \mathrm{~K} \leqslant T \leqslant 550 \mathrm{~K}$ ). Figure 2(a) demonstrates that all $\mathrm{CW}$ temperatures are positive, signalling predominantly FM exchange interactions. $\Theta$ increases linearly with $x$ in the $\mathrm{O}^{\prime}$ phase but is almost constant in the $\mathrm{O}$ and $\mathrm{R}$ phases. This behaviour cannot be explained by the enhancement of DE upon hole doping. The linear increase of $\Theta$ in the $\mathrm{O}^{\prime}$ phase on increasing $x$ rather provides clear experimental evidence that the longrange JT distortion suppresses FM exchange. The distortion is strong at $x=0$, revealing a corresponding small $\Theta$ value which results as an average from the anisotropic SE interactions (FM in the $a b$-plane, AFM along $c$ ). Upon doping, the JT distortion becomes weaker and the orbital degeneracy is more and more completely restored, a fact that leads to isotropic ferromagnetic SE. Concomitantly, $\Theta$ grows and finally recovers its value for the undistorted $\mathrm{O}$ phase at $x=0.15$. The $\mathrm{CW}$ temperatures become further enhanced in the $\mathrm{R}$ phase, as the buckling of the $\mathrm{MnO}_{6}$ octahedra becomes smaller, bringing the bond angles close to $180^{\circ}$ and increasing the $\mathrm{Mn}-\mathrm{O}-\mathrm{Mn}$ superexchange. It is puzzling that on the basis of our results the CW temperatures signal hole-independent FM exchange interactions in the R and especially in the O phase. Obviously, the hole-dependent DE interactions play only a negligible role at these low doping levels, a fact that also explains the semiconducting behaviour of the electrical resistance in these phases. As we discuss later, this FM exchange is derived from SE interactions between sites with orbital degeneracy and strong Hund's coupling.

Figure 2(b) shows the concentration dependence of the effective PM moments $\mu_{e f f}$, again for the different structural phases (see also table 1). The close correlation of $\mu_{\text {eff }}$ with the structural properties and hole doping is evident. For the R phase we indicate the PM moments 


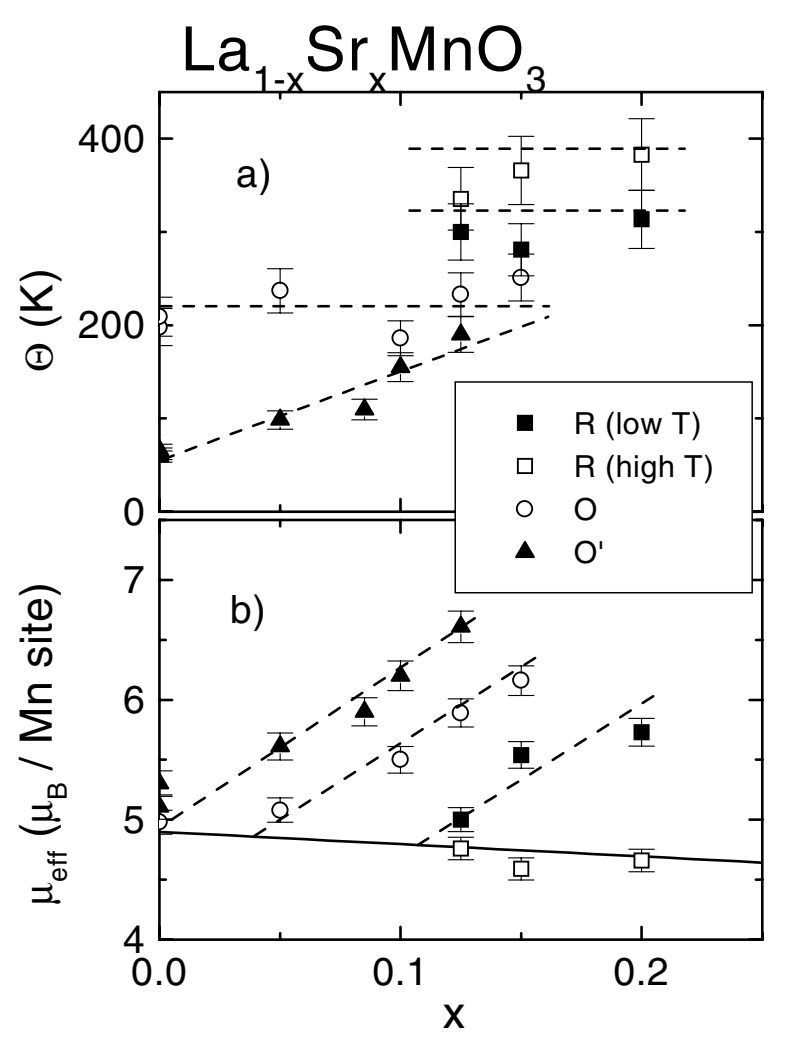

Figure 2. (a) Paramagnetic Curie-Weiss temperature $\Theta$ versus Sr concentration $x$ for the various structural phases. (b) The doping dependence of the effective paramagnetic moments. Dashed lines are guides to the eyes.

which result from an analysis of the susceptibility, either just above the structural or magnetic phase transitions (full squares), or of the high-temperature regime (empty squares). The latter are rather close to the calculated spin-only values for the mixed compounds (solid line). The PM moments for increased hole doping are highly enhanced, yielding values of almost $7 \mu_{B}$. These high values and the strong increase with hole doping reveal the importance of FM clusters within the PM phases. From these results, it follows that the clusters increase in size with increasing $x$, being largest close to the magnetic ordering temperatures. Short-rangeorder (SRO) effects are well known in FM metals, and experimentally are evidenced by a temperature dependence of the susceptibility as $\chi^{-1} \propto\left(T-T_{C}\right)^{\gamma}$, with $\gamma=4 / 3$. We tried to fit the experimental results for $x=0.2$ accordingly. The result is shown in the inset of figure 1 . The data can be described well taking $T_{C}=306 \mathrm{~K}$ and using an exponent of $\gamma=1.2$. This value is close to the one reported by Ghosh et al [24] for $\mathrm{La}_{0.7} \mathrm{Sr}_{0.3} \mathrm{MnO}_{3}$.

3.1.2. Susceptibility and magnetization. A detailed report on magnetic susceptibility and magnetization experiments has been given elsewhere [25]. Here we would like to report new results, specifically on the anisotropy of the magnetization. For the discussion of the groundstate properties it is useful to discuss the canted $(x<0.1)$ and the FM regime $(0.1 \leqslant x \leqslant 0.15)$ separately. We recall that both phases are insulating. 


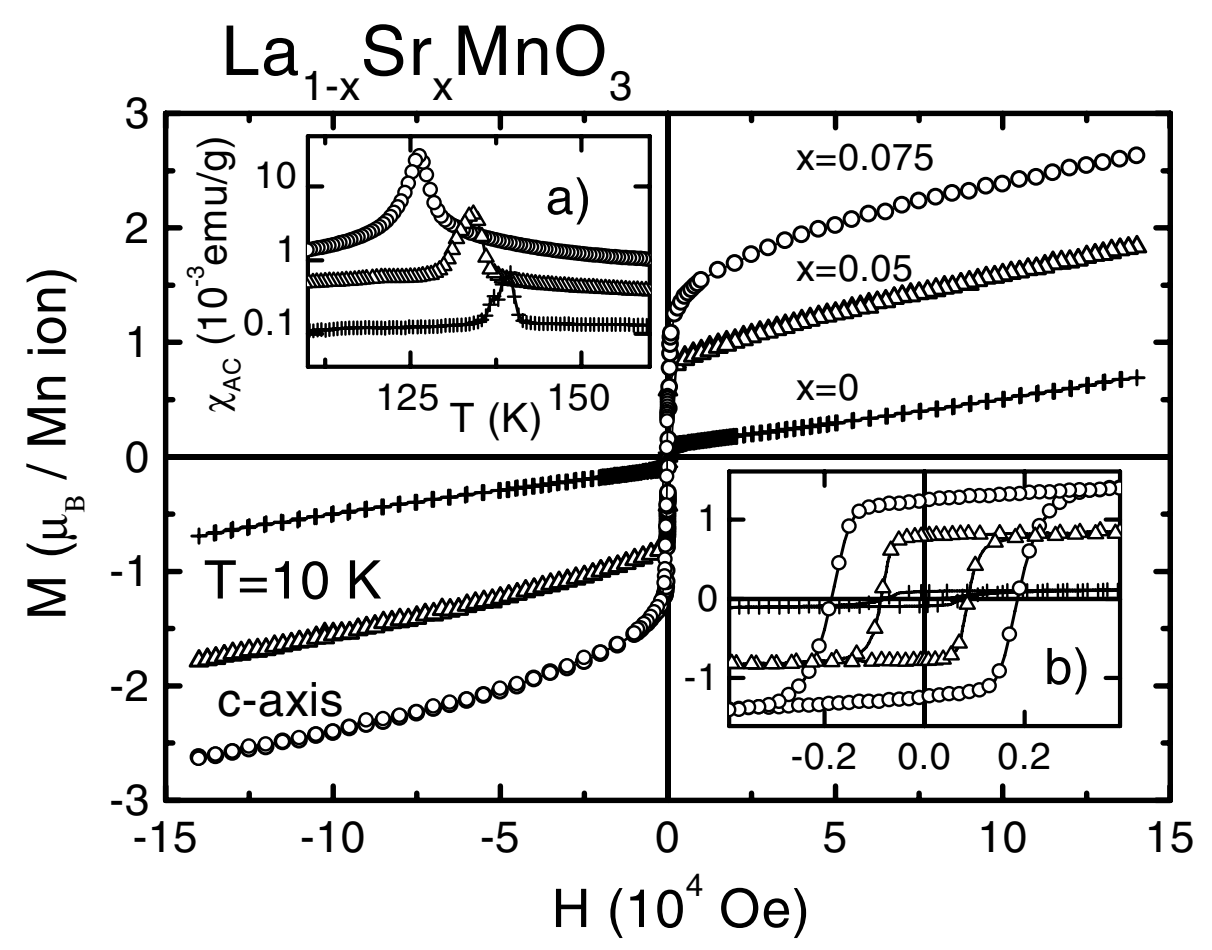

Figure 3. Magnetization versus magnetic field in $\mathrm{La}_{1-x} \mathrm{Sr}_{x} \mathrm{MnO}_{3}$ for concentrations $x=0$, 0.05 , and 0.075 at $10 \mathrm{~K}$. Inset (a) shows the ac susceptibility versus temperature for the same concentrations. Inset (b) gives an expanded view of the hysteresis behaviour of the magnetization curves.

3.1.2.1. $x<0.1$ : canted structure versus phase separation. The susceptibility in this lowdoping regime reveals one anomaly at the PM-to-AFM phase boundary [25]. Inset (a) in figure 3 gives the main result: at $T_{\mathrm{CA}}$, clearly AFM-like anomalies show up at the magnetic phase boundary. The phase transition temperatures shift to lower temperatures as the hole concentration increases, indicating the significant reduction of the AFM interactions between FM planes. This reduction is obviously correlated with the reduction of the long-range JahnTeller distortion as indicated by a strong decrease of the structural phase transition $T_{\mathrm{OO}^{\prime}}$ on increasing $x$.

The main frame of figure 3 shows the magnetization at $10 \mathrm{~K}$ for various compositions $(x<0.1)$ as functions of an external magnetic field. Here we tried to orient the $c$-axis of the samples parallel to the external field. However, most of the samples are strongly twinned and a perfect orientation is impossible. At very low fields we find hysteresis loops with a spontaneous magnetization that increases with increasing $x$ (see also inset (b) of figure 3). However, this spontaneous FM magnetization is followed by a linear increase of the magnetization $M$ on increasing the external fields. There is no sign of saturation even at an applied field of $140 \mathrm{kOe}$. This behaviour corresponds to the predictions of de Gennes [4] for the manganites in the CA phase. The basic effect is that the external magnetic field enforces a continuous reduction of the canting angle. In addition, the initial value of the spontaneous FM magnetization increases with $x$, a fact that directly demonstrates the reduction of the canting angle on hole doping. However, there is a common belief that the canted phase is unstable against electronic phase separation [26-29]. 
The phase-separation scenarios in the CA phase suggest that the itinerant charge carriers form ferromagnetic metallic droplets within the AFM and insulating ground states. The possibility that at low doping levels the holes form localized states has been discussed already by de Gennes [4], and the possibility of the formation of an inhomogeneous state has been further elucidated by Khomskiı̌ and Sawatzky [26]. Theoretically possible electronic phaseseparation scenarios have been put forward by Gorkov [27], Nagaev et al [28], and, on the basis of computational studies, Dagotto and co-workers [29]. We clearly have to state that on the basis of the susceptibility and magnetization measurements reported in figure 3 we cannot decide whether we have a canted or a mixed phase (i.e. phase separation). But recently Kumagai et al [30], on the basis of ${ }^{139} \mathrm{La}$ NMR data, have shown that the magnetic ground state of lightly doped $\mathrm{La}_{1-x} \mathrm{Sr}_{x} \mathrm{MnO}_{3}$ is microscopically homogeneous.

Here we would like to provide further experimental evidence that a canted phase with an average canting angle is the stable low-temperature ground state at least for $\mathrm{La}: \mathrm{SrMnO}_{3}$ for doping levels below 10\%. Figure 4 shows the anisotropy of the susceptibility $\left(T>T_{\mathrm{CA}}\right)$ and of the magnetization $\left(T<T_{\mathrm{CA}}\right.$ ) for $\mathrm{La}_{0.05} \mathrm{Sr}_{0.95} \mathrm{MnO}_{3}$. In X-ray experiments and in polarizationdependent antiferromagnetic resonance experiments [31] this small sample was characterized as an untwinned single crystal. The PM susceptibility is completely isotropic and follows a Curie-Weiss type of behaviour with a CW temperature of $112 \mathrm{~K}$ and a PM moment of $5.5 \mu_{B}$, values which fit well into the results presented in table 1 . The magnetization as observed at low fields ( $H<1 \mathrm{Oe}$ ) is strongly anisotropic, being close to zero along $\boldsymbol{a}$ and $\boldsymbol{b}$ but with a FM moment along the $c$-axis. Hysteresis loops taken at $10 \mathrm{~K}$ (figure 5) reveal that this anisotropy holds up to $140 \mathrm{kOe}$ and indicate clear FM hysteresis (with a coercive field along $c$ of $H_{c o} \approx 1000 \mathrm{Oe}$ ). The magnetic structure of $\mathrm{LaMnO}_{3+\delta}$ has been investigated by neutron

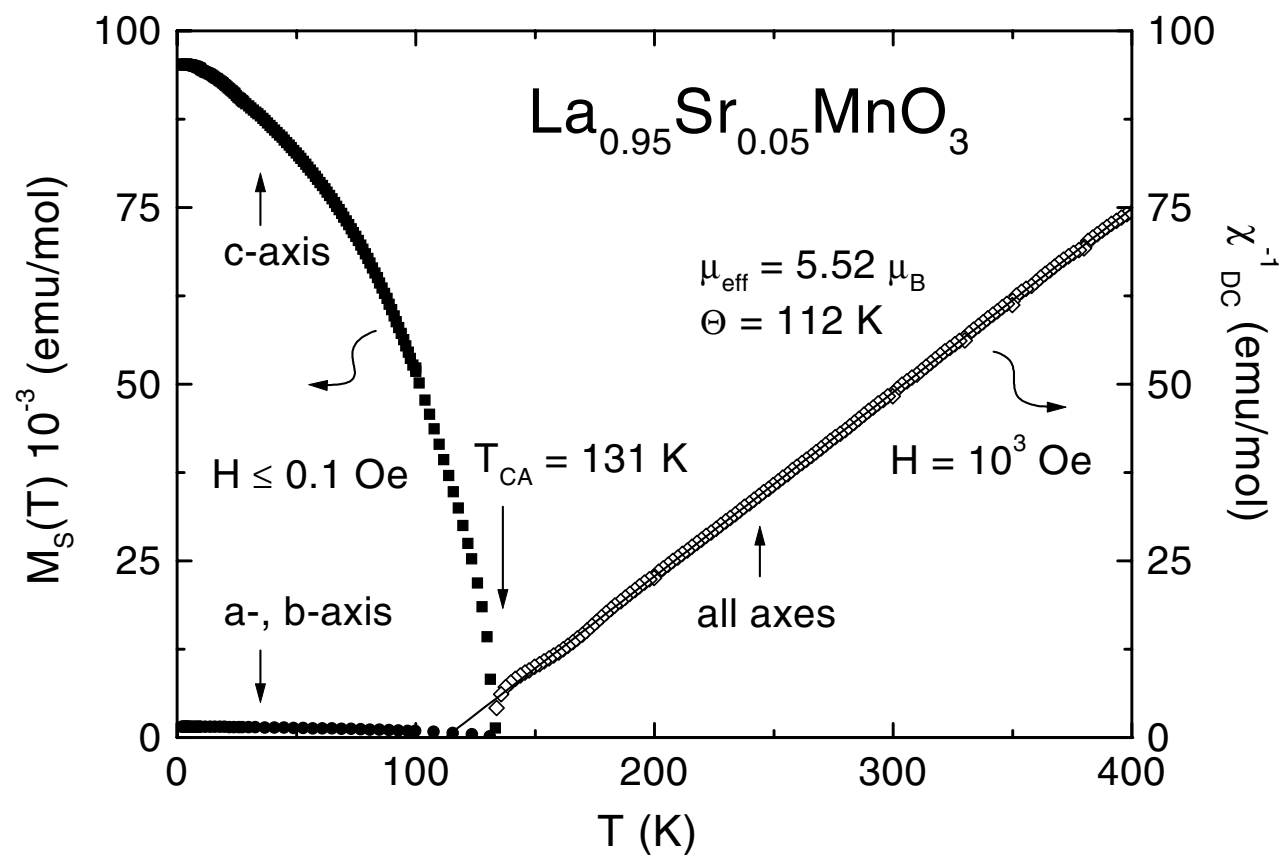

Figure 4. Temperature dependences of the inverse susceptibility $\chi^{-1}$ and the spontaneous magnetization $M_{s}$ along different orientations in $\mathrm{La}_{0.95} \mathrm{Sr}_{0.05} \mathrm{MnO}_{3}$. The inverse susceptibility was fitted according to Curie-Weiss behaviour (solid line). 


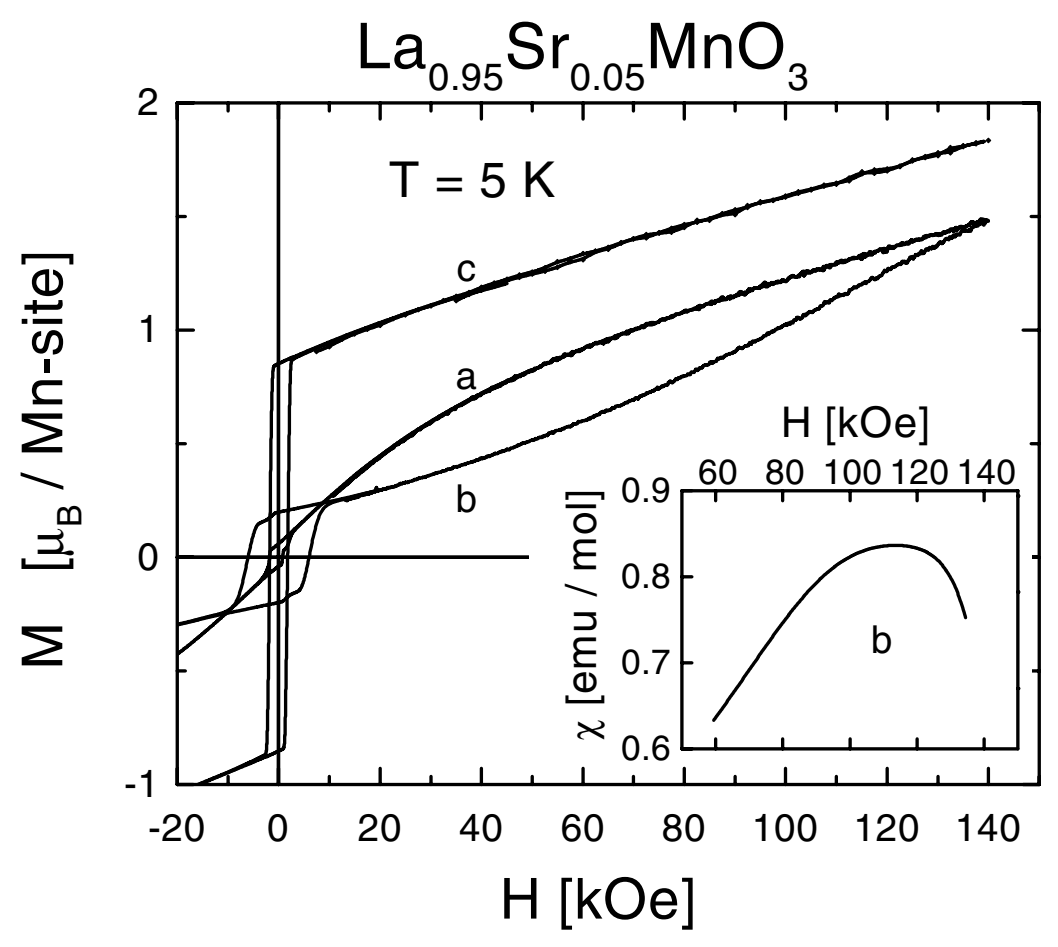

Figure 5. Magnetization versus magnetic field along the $a$-, $b$-, and $c$-directions at $5 \mathrm{~K}$ in $\mathrm{La}_{0.95} \mathrm{Sr}_{0.05} \mathrm{MnO}_{3}$. The inset shows the derivative of the magnetization along the $a$-axis.

diffraction and was characterized as layer-type (A-type) antiferromagnetic structure with FM coupling within the $a b$-planes and AF coupling between the $a b$-layers [2,21]. Due to spin canting, a spontaneous FM moment arises in the $c$-direction and coexists with the AF moment which is aligned in the $b$-direction. By applying an external field, additional magnetization is induced along all axes. The non-linear increase of the induced magnetization for $\boldsymbol{H} \| \boldsymbol{b}$ is due to the spin flop accompanied by the rotation of the underlying A-type spin structure. The derivative of the magnetization curve along $\boldsymbol{b}$ shows a broad maximum around $H=115 \mathrm{kOe}$ (see the inset of figure 5), as should be expected from a smeared-out spin-flop scenario. The spin-flop field in pure $\mathrm{LaMnO}_{3}$ is reported to be $H_{c} \approx 210 \mathrm{kOe}$ [32].

The observation of this strong magnetic anisotropy can hardly be explained by electronic phase-separation scenarios, e.g. assuming small ferromagnetic droplets within an AFM background. The DE-driven FM state in such hole-rich droplets is expected to be magnetically isotropic, as it is known to be for the purely FM phase in more highly doped compounds, e.g. for $x \approx 0.2$. Thus we believe that FM droplets would show an almost isotropic magnetization. On the other hand, the results can be naturally explained with a canted structure with the FM moment along $c$. Of course we are aware that our sample is insulating with no itinerant charge carriers. Hence all holes must be localized. But we recall de Gennes' deep discussion of a dilute assembly of Zener's bound electrons resulting in an overall behaviour very similar to that predicted for free carriers [4].

3.1.2.2. $x>0.1$. In order to study the complicated phase diagram for $0.1<x<0.175$, we performed a series of magnetization and susceptibility measurements. It is now well established 
that the low-temperature ground state in this concentration regime is a ferromagnetic insulator $[20,25]$ in an orthorhombic phase $\mathrm{O}^{\prime \prime}$ which reveals almost pseudocubic lattice constants. This $\mathrm{O}^{\prime \prime}$ phase most probably reveals charge (polaron) order $[17,18,33]$. The $\mathrm{O}^{\prime \prime} / \mathrm{FM} / \mathrm{I}$ phase is stable only below $200 \mathrm{~K}$ and is followed by a phase with different structure and different magnetic order at higher temperatures. For $x=0.125$, Endoh et al proposed a second FM and metallic phase. We have demonstrated that this intermediate phase is also insulating [25]. In figure 6 we show a series of susceptibility measurements for $x=0.1,0.125,0.15,0.175$, and 0.2. The absolute values for the large susceptibilities are strongly dependent on the demagnetization factors of the samples, which are not known precisely. However, the overall behaviour certainly is correct. For $x=0.1$, we still find two well defined phase transitions. The anomaly at $150 \mathrm{~K}$ is still reminiscent of that for $T_{\mathrm{CA}}$ as observed for $x<0.1$, and is followed by a second phase transition which establishes FM order in a new orthorhombic structure. For $x=0.125$, the same sequence of phase transitions seems to be present. However, for $x=0.15$, both anomalies are significantly broadened and smeared out, yielding one broad hump only. Finally, for $x=0.175$ and 0.2 , the strong increase of the susceptibility at high temperatures signals the onset of ferromagnetism. The downturn at lower temperatures indicates the phase transition from the orthorhombic $(\mathrm{O})$ to the rhombohedral $(\mathrm{R})$ phase, which does not change

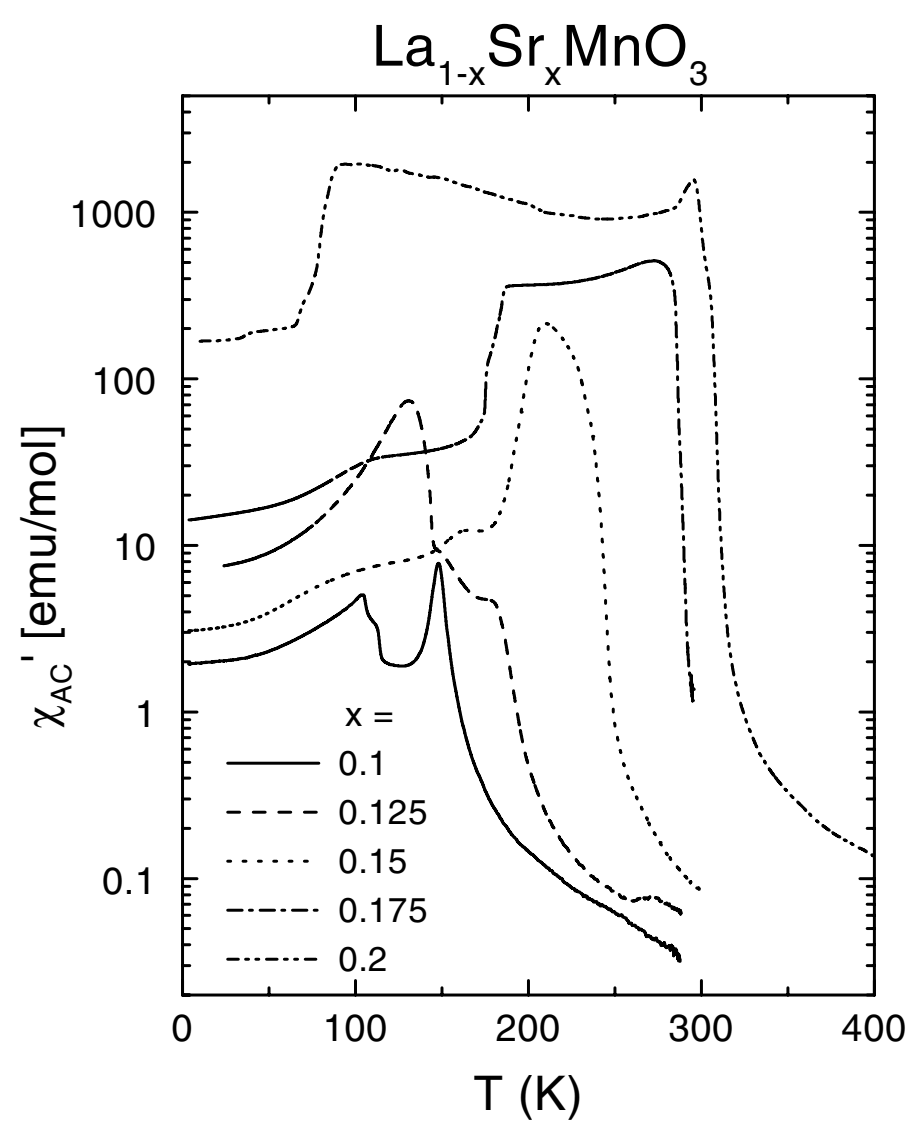

Figure 6. The ac susceptibility versus temperature for $\mathrm{La}_{1-x} \mathrm{Sr}_{x} \mathrm{MnO}_{3}$ for concentrations $x=0.1$, $0.125,0.15,0.175$, and 0.2 . The data were measured at frequencies between 0.3 and $3 \mathrm{kHz}$ with a stimulus of $H_{a c}=0.2 \mathrm{Oe}_{\mathrm{rms}}$. 
the ferromagnetic nature of the system.

Figure 7 shows a series of magnetization experiments. Focusing on the sample with a strontium concentration $x=0.1$, it is clear that the canted phase at elevated temperatures is followed by a structural transition into the $\mathrm{O}^{\prime \prime} / \mathrm{FM} / \mathrm{I}$ ground state, which can be induced by magnetic field (figure 7(a)). For $T>T_{\mathrm{CA}}=150 \mathrm{~K}$, the magnetization curves reveal the signature of a typical PM. Below $T_{\mathrm{CA}}$ we observe a linear increase of the magnetization on increasing field, characteristic for a CA phase. However, at a given critical field the magnetization jumps to higher values and becomes saturated. A closer inspection reveals two subsequent transitions, probably resulting from the twinning of our crystal. At these jumps the sample undergoes a magnetic transition into a FM state reaching full magnetization. From figure 7(b) we conclude that minor anomalies for $x=0.125(151 \mathrm{~K})$ and $x=0.15(217 \mathrm{~K})$ still signal this field-induced transition. We clearly have to admit that this small hysteresis loop close to fields of $1 \mathrm{~T}$ is our only experimental evidence that the high-temperature magnetic phase for $x=0.15$ is a canted phase.

The solid lines in figure 7 are fitted employing a simple Brillouin function containing a

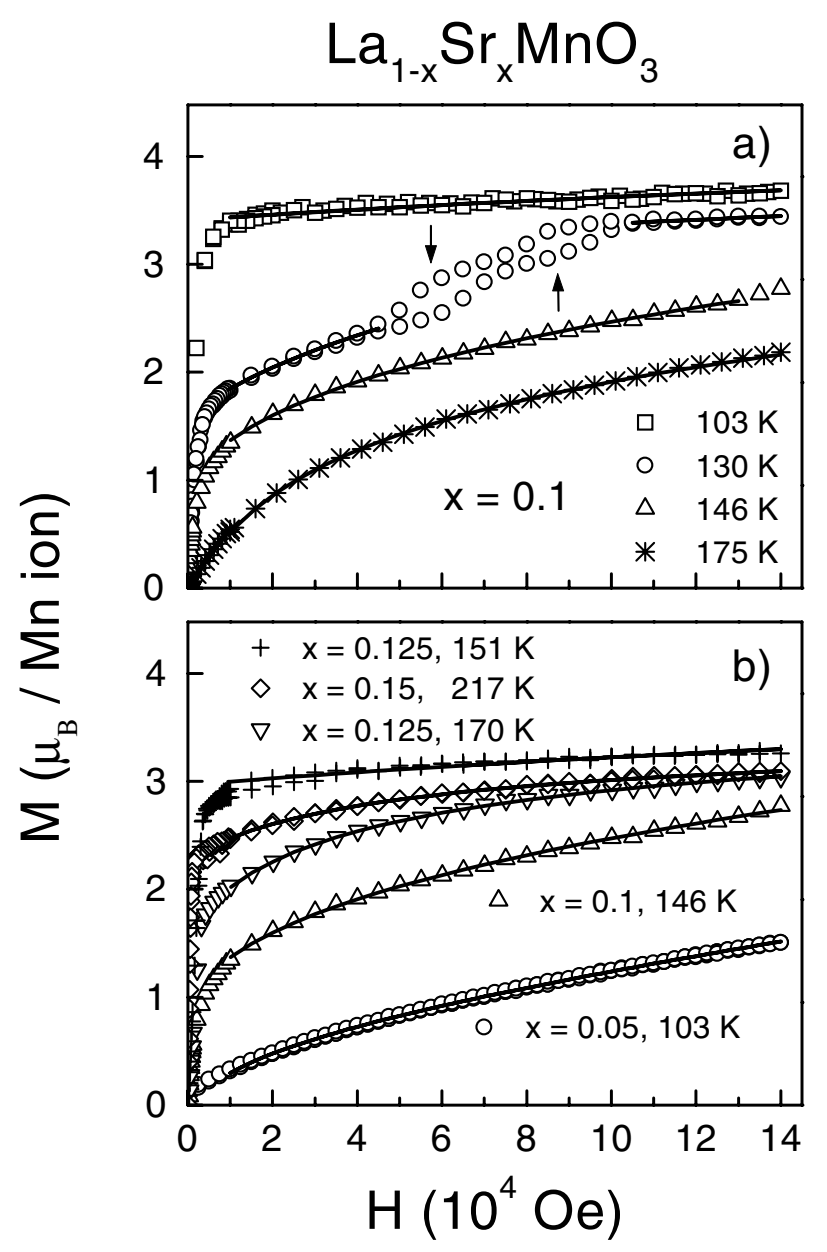

Figure 7. Isothermal magnetization versus field as observed in $\mathrm{La}_{0.9} \mathrm{Sr}_{0.1} \mathrm{MnO}_{3}$ at different temperatures (upper frame) and for concentrations $x=0.05,0.1,0.125$, and 0.15 (lower frame). 
mean interaction $\Theta^{\prime}$. The parameters of these fits are given in table 2. It is remarkable that the results obtained using this crude approximation agree with the expected values for the total spin $(S \stackrel{!}{=} 1.95$ for $x=0.1)$ and the saturation value $n S(n \stackrel{!}{=} 1)$ within $10 \%$ except for $x=0.125$ and 0.15 , in a narrow temperature regime above the $\mathrm{O}^{\prime \prime}$ phase (see the shaded area in figure 11, later). In this regime, much higher values for $S$ are needed to describe the experimental data. This may indicate the formation of an inhomogeneous cluster state in the crossover region between the $\mathrm{O}^{\prime} / \mathrm{I} / \mathrm{CA}$ phase and the $\mathrm{O} / \mathrm{M} / \mathrm{FM}$ phase.

Table 2. Parameters from the fits employing the Brillouin function of the hysteresis curves shown in figure 7. $\Theta^{\prime}$ models a mean interaction, $S$ is the total spin, and $n$ is the appertaining density of spins per unit cell.

\begin{tabular}{lllllrl}
\hline$x$ & $T(\mathrm{~K})$ & Structure & $\Theta^{\prime}(\mathrm{K})$ & $n$ & \multicolumn{1}{l}{$S$} & $n S$ \\
\hline 0.1 & 103 & $\mathrm{O}^{\prime \prime}$ & 157 & 1.0 & 2.1 & 2.1 \\
& 117 & $\mathrm{O}^{\prime \prime}$ & 189 & 1.0 & 2.0 & 1.9 \\
& 130 & $\mathrm{O}^{\prime \prime}$ & 183 & 1.0 & 2.0 & 2.0 \\
& 130 & $\mathrm{O}^{\prime}$ & 137 & 1.0 & 2.2 & 2.2 \\
& 146 & $\mathrm{O}^{\prime}$ & 148 & 1.2 & 1.8 & 2.1 \\
& 175 & $\mathrm{O}^{\prime}$ & 163 & 1.1 & 1.9 & 2.1 \\
\hline 0.125 & 151 & $\mathrm{O}^{\prime \prime}$ & 202 & 1.1 & 1.9 & 2.0 \\
& 170 & $\mathrm{O}^{\prime}$ & 191 & 0.064 & 26.5 & 1.7 \\
\hline 0.15 & 152 & $\mathrm{O}^{\prime \prime}$ & 228 & 1.1 & 1.9 & 2.0 \\
& 217 & $\mathrm{O}^{\prime}$ & 310 & 0.026 & 61.5 & 1.6 \\
\hline
\end{tabular}

\subsection{Electrical properties}

The ac and dc electrical properties of the single crystals used in the present investigations have been studied in detail and were published previously [34-36]. Here we want to focus on the hopping conductivity as observed at low doping levels. The temperature dependence of the electrical resistance for $x=0.1$ and 0.125 is plotted for various external magnetic fields in figure 8 . To demonstrate that the resistivity can be described by a three-dimensional variable-range-hopping (VRH) model [37] we use a $\log \rho$ versus $T^{-1 / 4}$ representation. At low temperatures $\left(T<T_{C}\right)$ the resistivity closely follows

$$
\rho=\rho_{0}\left(T / T_{0}\right)^{1 / 2} \exp \left[\left(T_{0} / T\right)^{1 / 4}\right]
$$

for all fields. Least-squares fitting of the experimental data for $T<T_{C}$ yields $T_{0}=2.3 \times 10^{8} \mathrm{~K}$, $\rho_{0}=1.6 \times 10^{-11} \Omega \mathrm{cm}$ for $x=0.1$ and $T_{0}=0.72 \times 10^{8} \mathrm{~K}, \rho_{0}=5.5 \times 10^{-8} \Omega \mathrm{cm}$ for $x=0.125$. The $T_{0}$ are rather typical of those observed for amorphous semiconductors and are in agreement with those reported by Fontcuberta et al [38]. For $x=0.1$ the resistivity can also be analysed for $T>T_{C}$ and again can be fitted using a VRH law with $T_{0}=1.72 \times 10^{8} \mathrm{~K}$, $\rho_{0}=1 \times 10^{-9} \Omega \mathrm{cm}$.

In figure 9 we report results from magnetoresistance experiments for $x=0.1,0.125$, and 0.15 . Here $[\rho(3 \mathrm{~T})-\rho(0 \mathrm{~T})] / \rho(0 \mathrm{~T})$ is plotted as a function of temperature. For $x=0.1$, the typical negative $\mathrm{CMR}$ around $T_{\mathrm{CA}}$ can be seen. It is followed by a strong positive MR indicating the transition into the FM and I pseudocubic $\mathrm{O}^{\prime \prime}$ phase at $T_{C}$ which is indeed more insulating than the $\mathrm{CA}$ phase. A strong shift towards higher temperatures of the transition $\mathrm{CA} / \mathrm{O}^{\prime} \rightarrow \mathrm{FM} / \mathrm{O}^{\prime \prime}$ is evident as the magnetic field is increased. Finally, below $T_{C}$ there is only a small field dependence of the resistivity, consistent with the almost saturated magnetization of the samples in the FM ground state. 


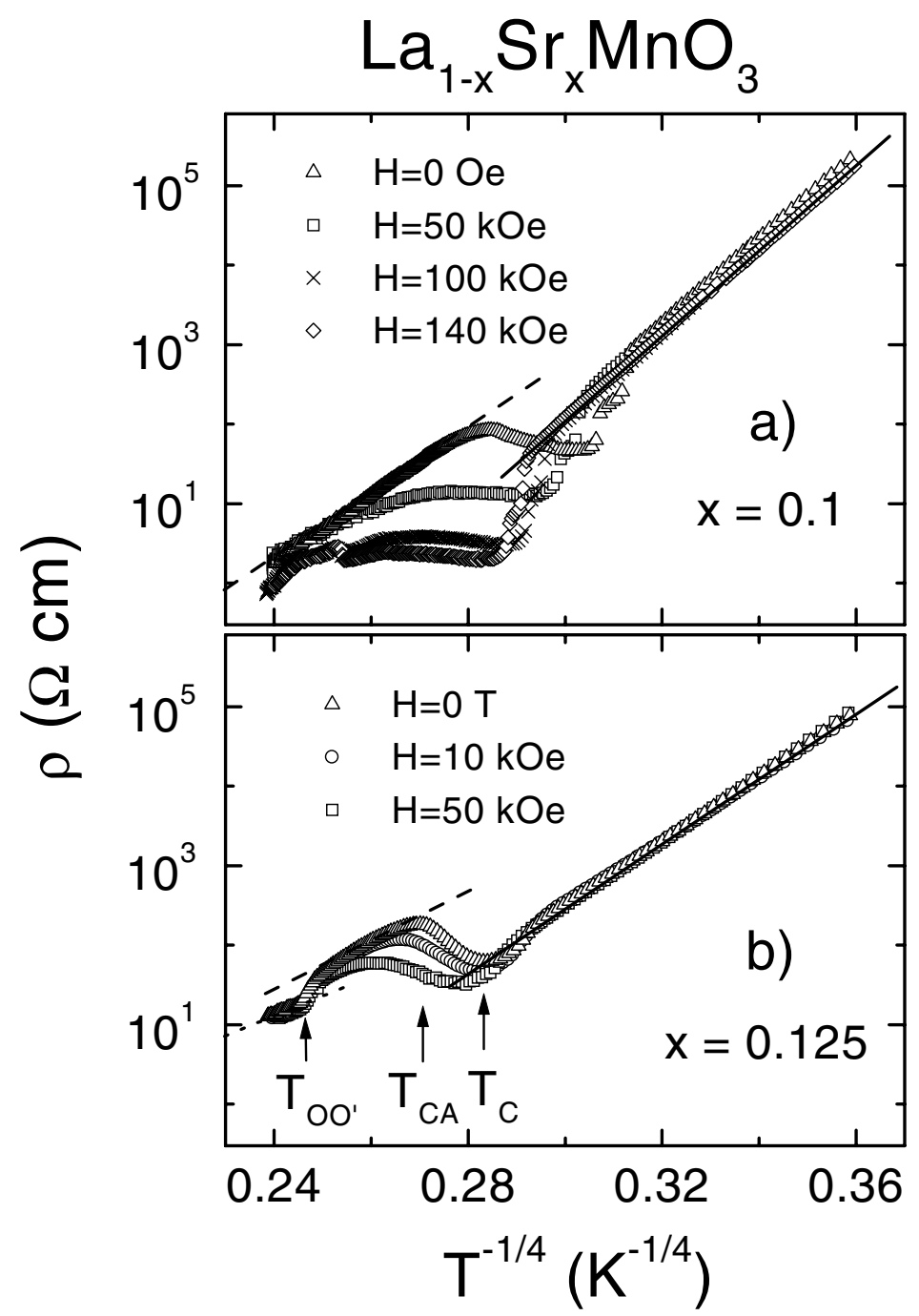

Figure 8. (a) The temperature dependence of the dc resistance for $x=0.1$ measured for various applied magnetic fields. (b) The same for $x=0.125$. Notice the $T^{-1 / 4}$-scaling of the temperature axis.

The relation of positive to negative MR changes considerably upon doping. For $x=0.15$, the transition into the FM and I ground state can hardly be detected and the response at all temperatures is dominated by the negative MR, in agreement with previous published results [15]. As discussed later, the positive MR signal is connected with the transition into a SE-driven FM/I phase. The ratio of positive to negative MR may be a measure of the relative importance of SE and DE.

In summary, the magnetoresistance measurements indicate a large positive MR at the transition $\mathrm{O}^{\prime} \mathrm{CA} \rightarrow \mathrm{O}^{\prime \prime} / \mathrm{FM}$, while negative MR effects appear close to the $\mathrm{O}^{\prime} / \mathrm{PM} \rightarrow \mathrm{O}^{\prime} / \mathrm{CA}$ and $\mathrm{O} / \mathrm{PM} \rightarrow \mathrm{O} / \mathrm{FM}$ phase boundaries. We note that positive MR effects in the vicinity of $x=1 / 8$ were also reported earlier [39-41]. 


\subsection{Elastic properties}

In order to get some idea about the role of structural degrees of freedom in the complex phase diagram of insulating low-doped manganite, the coupling between the lattice and the various order parameters was investigated for $\mathrm{Sr}$ concentrations lower than $x=0.175$ beyond which metallic behaviour occurs. Particularly in the vicinity of phase transitions, the elastic response to external stress depends sensitively on the lattice coupling and the symmetry of the order parameters involved. Ultrasonic experiments are therefore quite informative and may help to clarify whether the transitional behaviour in these systems is driven by lattice forces originating in the Jahn-Teller effect and orbital ordering, for instance.

Figure 10 shows preliminary results of sound velocity measurements performed on crystals with Sr contents of $x=0.05$ and 0.15 , and should be considered as an important completion of the elastic data obtained by Hazama et al [42] for $x=0.12$ and $x=0.165$. The most striking feature observed is a pronounced anomaly for longitudinal sound propagation along the $c$-direction $\left(c_{33}\right.$-mode) in crystalline samples with $x=0.15$. Since the singularity in

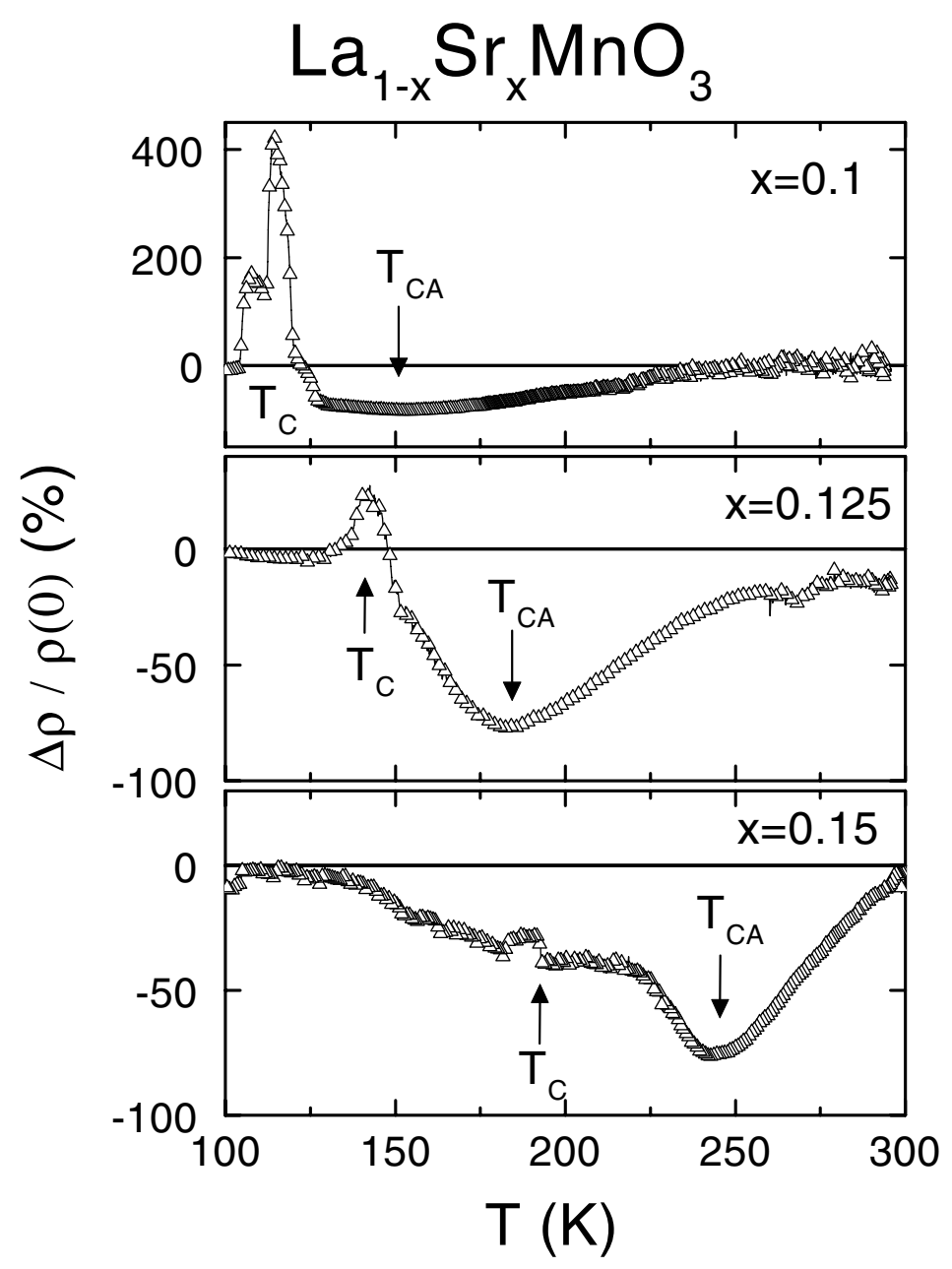

Figure 9. The MR of $\mathrm{La}_{1-x} \mathrm{Sr}_{x} \mathrm{MnO}_{3}$ for concentrations $x=0.1,0.125$, and 0.15 in an external field of $3 \mathrm{~T}$. 
the temperature-dependent sound velocity occurs close to $200 \mathrm{~K}$, it seems likely that one can attribute this anomaly to the structural transition from the orthorhombic $\mathrm{O}$ to the $\mathrm{O}^{\prime \prime}$ phase, although our results cannot rule out definitely the possibility of closely succeeding transitions $\mathrm{O} / \mathrm{O}^{\prime}$ and $\mathrm{O}^{\prime} / \mathrm{O}^{\prime \prime}$ at present. Remarkably, the sound velocity is strongly temperature dependent also below $200 \mathrm{~K}$ and one might speculate that the steep increase towards lower temperatures is mainly caused by domain formation upon cooling. On the other hand, the mean sound attenuation in the low-temperature phase is hardly larger than in the parent phase, which, however, gives strong support for the suggestion that the anomalously strong stiffening below the transition temperature is indeed an intrinsic property of the $\mathrm{O}^{\prime \prime}$ phase. According to figure 10 , the overall increase in the normalized sound velocity exceeds $10 \%$ (!) and is thus very reminiscent of the elastic behaviour due to charge-ordering phenomena, as found for instance in heavily doped $\mathrm{La}_{1-x} \mathrm{Ca}_{x} \mathrm{MnO}_{3}$ [43]. Upon cooling below ambient temperature, the lattice softening becomes significantly enhanced when passing through the magnetic transition at about $240 \mathrm{~K}$, as emphasized in figure 10 . This behaviour is in obvious contrast to the strong stiffening discussed above. All in all, the strong temperature dependence of the sound velocity in single-crystalline $\mathrm{La}_{0.85} \mathrm{Sr}_{0.15} \mathrm{MnO}_{3}$ confirms the assumption of structural degrees of freedom being heavily involved in the complex phase diagram.

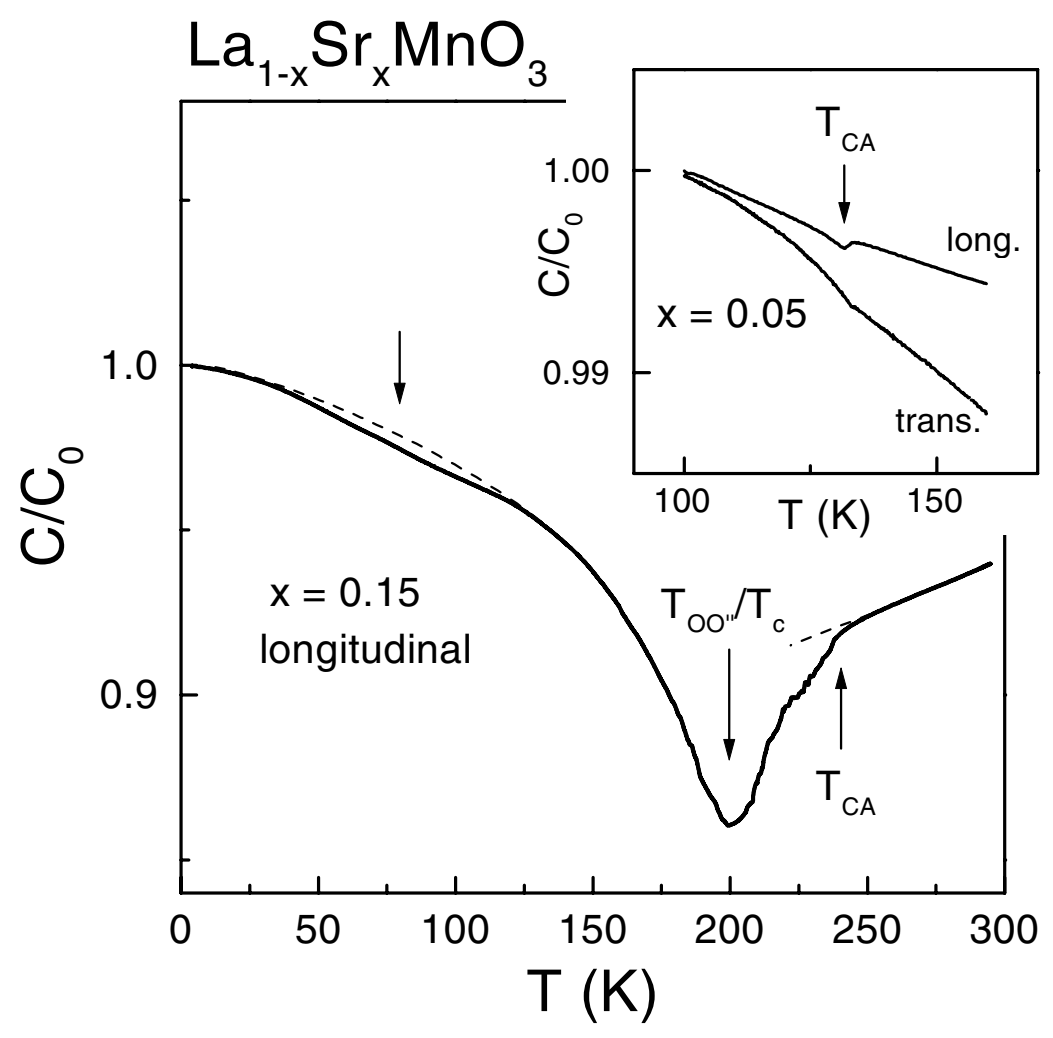

Figure 10. The temperature dependence of the normalized longitudinal sound velocity for $x=0.15$. Inset: the same for longitudinal and transverse modes for $x=0.05$.

The elastic behaviour of low-doped manganite with $x=0.05$ is completely different from the previous case as shown for comparison in the inset of figure 10. Here, the temperaturedependent sound velocities of both transverse and longitudinal sound show only rather small 
though distinct anomalies right at the transition temperature where antiferromagnetic order occurs. Obviously, the elastic properties change only marginally upon cooling from the PM to the CA phase, clearly pointing to an order parameter which is only weakly coupled to the lattice. Such a transitional behaviour, however, is quite common for many antiferromagnetic insulators, such as $\mathrm{MnF}_{2}$ [44], where elastic anomalies of the same order at the Néel temperature have to be ascribed to magnetostrictive effects. Hence, there is no hint from our sound data that orbital ordering plays any significant role in the antiferromagnetic transition of this low-doped manganite $(x<0.1)$.

Concerning the crystal with higher Sr doping, it should be further noted that a weak elastic anomaly has been resolved additionally at temperatures around $75 \mathrm{~K}$. Even though slight changes were observed in the magnetic susceptibility as well, and distinct anomalies are also reported for the thermopower [45] in this temperature range, no definite conclusions can be drawn at present about the physical origin of this phenomenon. However, bearing in mind that the Sr content is close to the critical concentration of the metal-insulator transition at $x=0.175$, the possibility of the occurrence of a two-phase regime cannot fully be excluded.

\subsection{Phase diagram}

On the basis of these experimental results, we constructed a structural, magnetic, and electronic phase diagram (figure 11). At low temperatures and low concentrations $(x<0.1)$, we find an insulating orthorhombic phase $\mathrm{O}^{\prime}$ which reveals canted antiferromagnetism. The phase diagram becomes much more complicated at higher $\mathrm{Sr}$ concentrations. Our results provide clear experimental evidence that for $0.1 \leqslant x \leqslant 0.15$, the ground state is a FM insulator, and that, for $x=0.1$ and $x=0.125$, this is followed by a canted spin state at elevated temperatures. Using neutron diffraction techniques, Argyriou et al [46] have monitored the temperature dependence of the canting angle $\theta$ for $x=0.125$ and observed a continuous decrease below $T_{\mathrm{CA}}$ and a lock-in-like phenomenon for the canting angle at approximately $20^{\circ}$ below $160 \mathrm{~K}$. On the basis of our data, we favour also a CA structure for $x=0.15$, but no definite conclusions can be drawn. At the $\mathrm{O}^{\prime}-\mathrm{O}^{\prime \prime}$ phase boundary which is coupled with the $\mathrm{CA} \rightarrow \mathrm{FM}$ transition, the resistivity steeply increases. For $x>0.17$, the ground state is a FM metal, revealing an $\mathrm{O}$ structure for $x<0.2$ and a $\mathrm{R}$ structure for $x>0.2$.

Taking into account the magnetic anisotropy for $x<0.1$, a canted structure appears to be more favourable than a phase-separation scenario. A ferromagnetic droplet within an antiferromagnetic background should yield no strongly anisotropic response. Additional experimental evidence from high-field AFMR measurements supports this picture of a canted state in the low-doped regime [31]. The I ground state for $0.1 \leqslant x \leqslant 0.15$ is FM with the full expectable saturation value of the magnetization. This implies that all $\mathrm{Mn}^{3+} / \mathrm{Mn}^{4+}$ spins contribute, and neither phase separation nor a relevant AFM canting component has to be considered. On the basis of our data, indications for an electronically phase-separated regime exist only for the shaded area of figure 11. It is in the regime close to the structural instability where the long-range JT distortion becomes suppressed and DE interactions start to play a more fundamental role.

The structural phase transitions have also been studied in detail using FIR techniques. Here we found strong temperature dependencies of the phonon modes and damping constants between the different magnetic and structural phase boundaries. This issue will be addressed in a separate publication together with our optical measurements [47].

The transition from the CA and JT-distorted $\mathrm{O}^{\prime}$ phase to the pseudocubic $\mathrm{O}^{\prime \prime}$ phase, which is intimately coupled to a transition into an insulating FM ground state, calls for a different picture, as it can be given by the interplay of JT distortions and DE interactions alone. For undoped 


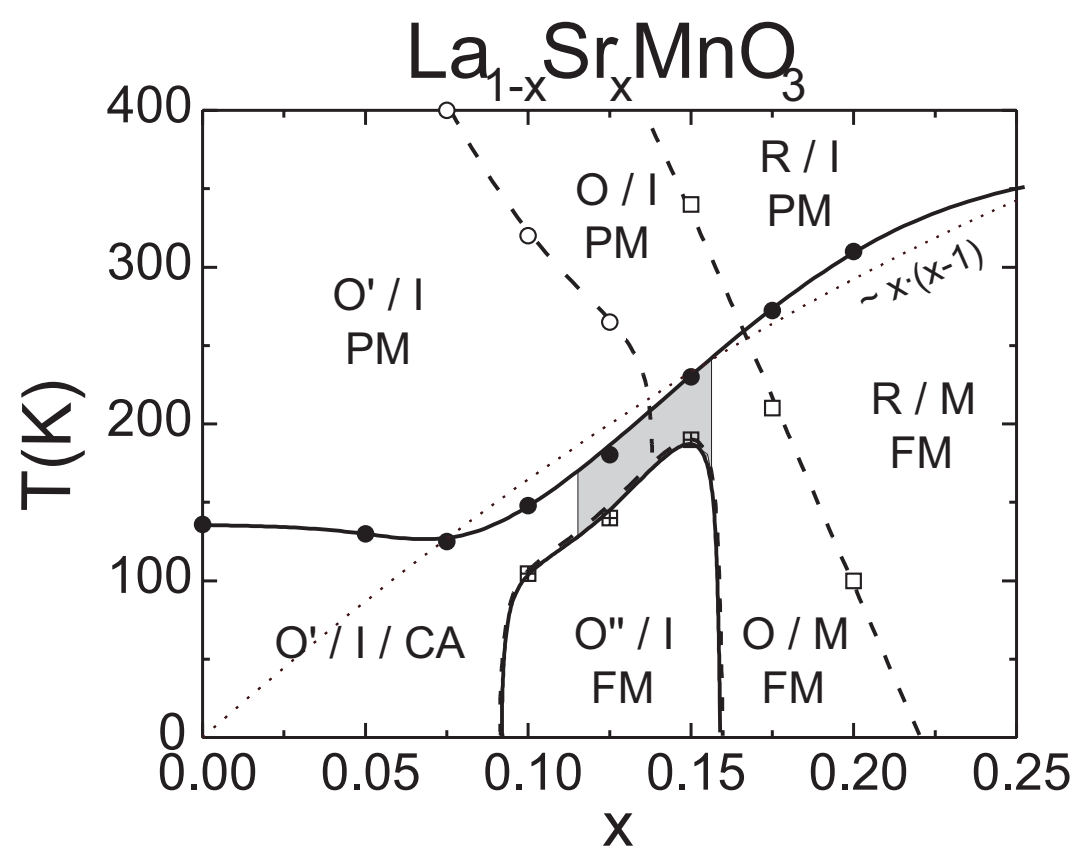

Figure 11. The $(x, T)$ phase diagram of $\mathrm{La}_{1-x} \mathrm{Sr}_{x} \mathrm{MnO}_{3}$. The structural $\left(\mathrm{O}, \mathrm{O}^{\prime}, \mathrm{O}^{\prime \prime}, \mathrm{R}\right)$, magnetic (PM, CA, FM), and electronic (M, I) phases are indicated. Open symbols (dashed lines) denote structural phase boundaries; solid symbols (full lines) denote magnetic phase boundaries.

$\mathrm{LaMnO}_{3}$ the magnetic properties can be understood taking only the predominant JT distortion of the $\mathrm{MnO}_{6}$ octahedra into account. The double degeneracy of the $\mathrm{e}_{\mathrm{g}}$ orbitals is lifted by a long-range cooperative Jahn-Teller distortion resulting in an ordering of the $\mathrm{d}_{3 r^{2}-z^{2}}$ orbitals, as argued by Goodenough [48] and Solovyev et al [49] on the basis of theoretical considerations and recently confirmed experimentally by Murakami et al [50]. As a consequence of this JTdriven orbital ordering, the A-type AFM state is established below $T_{N}$. A small intrinsic canting in $\mathrm{LaMnO}_{3}$ originates from the interplay between the Dzyaloshinsky-Moriya interaction [51] and the lattice distortion [49].

The possibility of orbital ordering in transition metal oxides due to exchange interactions different to that resulting from JT distortions [52] was first pointed out by Roth [53] and has been extensively studied by Kugel and Khomskir [54]. It has been shown that in the case of orbital degeneracy two transitions take place, one into an orbitally ordered state and a second into a spin-ordered state, both driven by SE mechanisms. This SE differs from the ordinary one due to the fact that each electron has four degrees of freedom: two orbital states $\left(\mathrm{d}_{3 r^{2}-z^{2}}, \mathrm{~d}_{x^{2}-y^{2}}\right)$ and two spin states (spin up, spin down). The presence of intra-atomic exchange (Hund's rule coupling) produces ferromagnetism below the orbital-ordering phase transition [55]. A modern view of these problems has been recently considered by Held and Vollhardt [56]. We remark that in the $\mathrm{O}^{\prime}$ phase the degeneracy of the $\mathrm{Mn}^{3+} \mathrm{e}_{\mathrm{g}}$ orbitals is lifted by the JT distortion, yielding anisotropic SE interactions, which are FM in-plane and AFM out-of-plane [49]. On the other hand, in the pseudocubic $\mathrm{O}^{\prime \prime}$ phase where the degeneracy is restored, the SE interactions become FM and isotropic.

The phase diagram for $x<0.1$ can be explained assuming a JT-derived $\mathrm{O}^{\prime}$ phase with strongly anisotropic $\mathrm{Mn}-\mathrm{O}$ bonds. Dominating anisotropic SE interactions establish a canted phase which is directly derived from the A-type AFM of the parent compound. We do not 
think that the canting results directly from DE interactions, since the induced carriers are localized [36] and hence cannot mediate a homogeneous canted state throughout the whole lattice. An alternative explanation can be given if one assumes that the induced holes become trapped and polarize the orbitals of the surrounding Mn sites, leading to the formation of FM clusters (see section 3.1.1) embedded in a PM matrix. If the carriers are bound or nearly bound, the distortion becomes non-homogeneous but the average effects are similar $[4,26,57]$. Of course it is intriguing to speculate that this FM short-range order (SRO) may be the precursor of electronic phase-separation effects. One may argue that these FM clusters could easily survive the magnetic phase transition at $T_{\mathrm{CA}}$, yielding FM regions in an AFM background. However, in this framework it is quite difficult to understand why the FM spontaneous moment and the AFM ordering appear at the very same temperature (i.e. $T_{\mathrm{CA}}$ ). This point still remains controversial, and more theoretical and experimental work is needed. The decrease of $T_{\mathrm{CA}}(x)$ in this regime results from a continuous suppression of the JT distortions with increasing hole doping and a concomitant weakening of the anisotropic SE. On the other hand, the increase of $T_{\mathrm{CA}}(x)$ upon doping for $0.1 \leqslant x \leqslant 0.15$ signals that the magnetic state is now driven by 3D ferromagnetic DE interactions which are perturbed by weak antiferromagnetic SE interactions, yielding a CA state. An increase of the hopping matrix elements due to enhanced DE interactions is evidenced by the decrease in resistivity at $T_{\mathrm{CA}}$, which is not present for the $x<0.1$ samples [15,35]. However, the DE mechanism is not strong enough to create a metallic phase. It has been demonstrated recently that for $0.1 \leqslant x \leqslant 0.15$ the regime with $\mathrm{d} \rho / \mathrm{d} T>0$ is still insulating and is characterized by hopping of localized charge carriers [36].

It is obvious that the DE mechanism cannot explain the FM and insulating ground state for $0.1 \leqslant x \leqslant 0.15$. We propose that ferromagnetism in this regime is driven by isotropic SE interactions within the insulating pseudocubic phase $\mathrm{O}^{\prime \prime}$ as discussed above, and it is straightforward to see that $\mathrm{O}^{\prime \prime}$ probably indicates a new orbitally ordered phase. The structural phase transition which precedes the FM phase indicates a transition from a JT-derived orbital order $\left(\mathrm{O}^{\prime}\right.$ phase) to an exchange-derived orbital order $\left(\mathrm{O}^{\prime \prime}\right.$ phase $)$.

The fundamental open question is that of how the system overcomes the long-range JT distortion and establishes a new orbital order in a pseudocubic phase. The most plausible explanation is that at this point charge order (CO) plays an important role [17]. Recently, Ahn and Millis [58] argued that the interplay of charge and orbital order to minimize the lattice energy could be the origin of this ordering. However, it is astonishing that this $\mathrm{CO}$ state is stabilized by an external magnetic field, yielding a positive MR effect as shown in figure 9 . In other charge-ordered manganites (e.g. $\operatorname{Pr}_{1-x} \mathrm{Ca}_{x} \mathrm{MnO}_{3}$ ) the application of a magnetic field melts the $\mathrm{CO}$ and drives the system into a FM and metallic state. This rather counterintuitive behaviour of the MR signals that CO may not occur in the present system (at least not in the vicinity of $x=0.12$ ). Nonetheless, these results demonstrate once more that the intimate coupling between spin, charge, and orbital degrees of freedom is not fully understood and deserves further attention.

\section{Conclusions}

We have studied the magnetic, transport, and elastic properties of the manganite system $\mathrm{La}_{1-x} \mathrm{Sr}_{x} \mathrm{MnO}_{3}$ for $x \leqslant 0.2$. For the paramagnetic phase we found experimental evidence for the occurrence of short-range ferromagnetic order. On hole doping, the Curie-Weiss temperatures increase in the Jahn-Teller-distorted $\mathrm{O}^{\prime}$ phase, but remain almost constant in the high-temperature $\mathrm{O}$ and $\mathrm{R}$ phases. It seems that in the phases with equal $\mathrm{Mn}-\mathrm{O}$ bond lengths, ferromagnetic superexchange interactions dominate at not-too-high hole concentrations. In the magnetoresistance, we found small negative MR effects around $T_{\mathrm{CA}}$ and strong positive 
effects at $T_{C}$. We were able to exclude the possibility of electronic phase separation for the CA phase for concentrations $x<0.1$. Finally, from the anomalies in the magnetic susceptibility, electrical resistance, and elastic measurements, a detailed phase diagram for this doping regime was obtained. We pointed out the importance of superexchange interactions for these compounds in the case of orbital degeneracy, and explained the nature of the ferromagnetic and insulating phase for $0.1 \leqslant x \leqslant 0.15$.

\section{Acknowledgments}

We acknowledge valuable discussions with K Samwer and K Held. This work was in part supported by the BMBF under contract No 13N6917/Elektronische Korrelationen und Magnetismus.

\section{References}

[1] Jonker G H and van Santen J H 1950 Physica 16337

[2] Wollan E O and Koehler V C 1955 Phys. Rev. 100545

[3] Zener C 1951 Phys. Rev. 82403

[4] de Gennes P G 1960 Phys. Rev. 118141

[5] Kusters R M, Singleton J, Keen D A, McGreevy R and Hayes W 1989 Physica B 155362

[6] Chahara K, Ohno T, Kasai M and Kozono Y 1993 Appl. Phys. Lett. 631990 von Helmolt R, Wecker J, Holzapfel B, Schulz L and Samwer K 1993 Phys. Rev. Lett. 712331

Jin S, Tiefel T H, McCormack M, Fastnacht R A, Ramesh R and Chen L H 1994 Science 264413

[7] Furukawa N 1994 J. Phys. Soc. Japan 633214

[8] Millis A J, Littlewood P B and Shraiman B I 1995 Phys. Rev. Lett. 745144

[9] Röder J H, Zang J and Bishop A R 1996 Phys. Rev. Lett. 761356

[10] Maezono R, Ishihara S and Nagaosa N 1998 Phys. Rev. B 5811583

[11] Kilian R and Khaliullin G 1998 Phys. Rev. B 58 R11 841

[12] Horsch P, Jaklič J and Mack F 1999 Phys. Rev. B 596217

[13] van den Brink J, Horsch P, Mack F and Oleś A 1999 Phys. Rev. B 596795

[14] Bogush A K, Pavlov V I and Balyko L V 1983 Cryst. Res. Technol. 18589

[15] Urushibara A, Moritomo Y, Arima T, Asamitsu A, Kido G and Tokura Y 1995 Phys. Rev. B 5114103

[16] Kawano H, Kajimoto R, Kubota M and Yoshizawa H 1996 Phys. Rev. B 532202 Kawano H, Kajimoto R, Kubota M and Yoshizawa H 1996 Phys. Rev. B 53 R14 709

[17] Yamada Y, Hino O, Nohdo S and Kanao R 1996 Phys. Rev. Lett. 77904

[18] Zhou J-S, Goodenough J B, Asamitsu A and Tokura Y 1997 Phys. Rev. Lett. 793234

[19] Vasiliu-Doloc L, Lynn J W, Moudden A H, de Leon-Guevara A M and Revcolevschi A 1998 Phys. Rev. B 58 14913

[20] Endoh Y, Hirota K, Ishihara S, Okamoto S, Nishizawa A, Fukuda T, Kimura H, Nojiri H, Kaneko K and Maekawa S 1999 Phys. Rev. Lett. 824328

[21] Hennion M, Moussa F, Biotteau G, Rodriguez-Carvajal J, Pinsard L and Revcolevschi A 1998 Phys. Rev. Lett. 811957

Hennion M, Moussa F, Biotteau G, Rodriguez-Carvajal J, Pinsard L and Revcolevschi A 1999 Preprint condmat/9910361

[22] Miller J G and Bolef D I 1969 Rev. Sci. Instrum. 40915

[23] Töpfer J and Goodenough J B 1997 J. Solid State Chem. 130117

[24] Ghosh K, Lobb C J, Greene R L, Karabashev S G, Shulyatev D A, Arsenov A A and Mukovskii Y 1998 Phys. Rev. Lett. 814740

[25] Paraskevopoulos M, Mayr F, Hartinger C, Pimenov A, Hemberger J, Lunkenheimer P, Loidl A, Mukhin A A, Ivanov V Yu and Balbashov A M 2000 J. Magn. Magn. Mater. 211118

[26] Khomskiĭ D I and Sawatzky G A 1997 Solid State Commun. 10287 and references therein

[27] Gorkov L P 1998 Sov. Phys.-Usp. 41589

[28] Nagaev E L 1996 Sov. Phys.-Usp. 39781

Nagaev E L 1998 Phys. Rev. B 582415

Nagaev E L 1998 Sov. Phys.-JETP 871214 
[29] Yunoki S, Moreo A and Dagotto E 1998 Phys. Rev. Lett. 815612

Moreo A, Yunoki S and Dagotto E 1999 Preprint cond-mat/9901057

[30] Kumagai K, Iwai A, Tomioka Y, Kuwuhara H, Tokura Y and Yakubovskii A 1999 Phys. Rev. B 5997

[31] Mukhin A A, Ivanov V Yu, Travkin V D, Pimenov A, Loidl A and Balbashov A M 2000 Europhys. Lett. 49514 Pimenov A, Biberacher M, Ivannikov D, Loidl A, Ivanov V Yu, Mukhin A A and Balbashov A M 2000 Preprint

[32] Mitsudo S, Hirano K, Nojiri H, Motokawa M, Hirota K, Nishizawa A, Kaneko N and Endoh Y 1998 J. Magn. Magn. Mater. 177-181 877

[33] Mizokawa T, Khomskiǔ D I and Sawatzky G A 1999 Preprint cond-mat/9912021

[34] Mukhin A A, Ivanov V Y, Travkin V D, Lebedev S P, Pimenov A, Loidl A and Balbashov A M 1998 JETP Lett. 68356

[35] Seeger A, Lunkenheimer P, Hemberger J, Mukhin A A, Ivanov V Y and Balbashov A M 1999 J. Phys.: Condens. Matter 113273

[36] Pimenov A, Hartinger C, Loidl A, Mukhin A A, Ivanov V Y and Balbashov A M 1999 Phys. Rev. B 5912419

[37] Mott N F and Davis E A 1979 Electronic Processes in Non-Crystalline Materials (Oxford: Oxford University Press)

[38] Fontcuberta J, Martinez B, Seffar A, Piñol S, Garcia-Muñoz J L and Obradors X 1996 Phys. Rev. Lett. 761122

[39] Uhlenbruck S, Teipen R, Klingeler R, Büchner B, Friedt O, Hücker M, Kierspel H, Niemöller T, Pinsard L, Revcolevschi A and Gross R 1999 Phys. Rev. Lett. 82185

[40] Senis R, Laukhin V, Martinez B, Fontcuberta J, Obradors X, Arsenov A A and Mukovskii Y M 1998 Phys. Rev. B 5714680

[41] Ghosh K, Greene R L, Lofland S E, Bhagat S M, Karabashev S G, Shulyatev D A, Arsenov A A and Mukovskii Y M 1998 Phys. Rev. B 588206

[42] Hazama H, Nemoto Y, Goto T, Asamitsu A and Tokura Y 2000 Physica B submitted

[43] Ramirez A P, Schiffer P, Cheong S-W, Chen C H, Bao W, Palstra T T M, Gammel P L, Bishop D J and Zegarski B 1996 Phys. Rev. Lett. 763188

[44] Melcher R L 1970 Phys. Rev. B 2733

[45] Uhlenbruck S, Büchner B, Gross R, Freimuth A, de Leon-Guevara A M and Revcolevschi A 1998 Phys. Rev. B 57 R5571

[46] Argyriou D N, Mitchell J F, Potter C D, Hinks D G, Jorgensen J D and Bader S D 1996 Phys. Rev. Lett. 763826

[47] Mayr F, Hartinger C, Paraskevopoulos M, Pucher K, Pimenov A, Hemberger J, Loidl A, Mukhin A A and Balbashov A M 2000 to be published

[48] Goodenough J B 1955 Phys. Rev. 100564

[49] Solovyev I, Hamada N and Terakura K 1996 Phys. Rev. Lett. 764825

[50] Murakami Y, Hill J P, Gibbs D, Blume M, Koyama I, Tanaka M, Kawata H, Arima T, Tokura Y, Hirota K and Endoh Y 1998 Phys. Rev. Lett. 81582

[51] Dzyaloshinsky I 1958 J. Phys. Chem. Solids 4241 Moriya T 1960 Phys. Rev. 12091

[52] Jahn H A and Teller E 1937 Proc. R. Soc. A 161220 Jahn H A 1938 Proc. R. Soc. A 164117

[53] Roth L M 1966 Phys. Rev. 149306 Roth L M 1967 J. Appl. Phys. 381065

[54] Kugel K I and Khomskiı̆ D I 1972 JETP Lett. 15446 Kugel K I and Khomskiǔ D I 1973 Sov. Phys.-JETP 37725

[55] Inagaki S 1975 J. Phys. Soc. Japan 39596

[56] Held K and Vollhardt D 1998 Eur. Phys. J. B 5473

[57] Kilian R and Khaliullin G 1999 Preprint cond-mat/9906045

[58] Ahn K H and Millis A J 1998 Phys. Rev. B 583697 